In press, Behaviour Research and Therapy

\title{
Effectiveness of Group Cognitive-Behavioral Treatment for Childhood Anxiety in Community Clinics
}

\author{
Wai-yee Lau ${ }^{\mathrm{a}}$ \\ Charlotte Kwok-ying Chan ${ }^{\mathrm{a}}$ \\ Johnson Ching-hong $\mathrm{Li}^{\mathrm{b}}$ \\ and \\ Terry Kit-fong $\mathrm{Au}^{\mathrm{c}}$
}

a: Child Assessment Service, Department of Health, 2/F, 147L Argyle Street, Kowloon, Hong Kong;

b: Department of Educational Psychology, Faculty of Education, University of Alberta, Edmonton, Alberta, T6G 2G5, Canada;

c: Department of Psychology, The University of Hong Kong, Pokfulam Road, Hong Kong.

Correspondence should be sent to:

Terry Kit-fong Au

Department of Psychology

The University of Hong Kong

Pokfulam Road, Hong Kong, China

Email: terryau@hku.hk

Tel: (852) 2859-2383

Fax: (852) 2858-3518 


\begin{abstract}
This study evaluated the effectiveness of cognitive-behavioral treatment for childhood anxiety in a community clinic setting in Hong Kong, China. Forty-five clinically-referred children (age 6 - 11 years) were randomly assigned to either a cognitive-behavioral treatment program or a waitlist-control condition. Children in the treatment condition showed significant reduction in anxiety symptoms — both statistically and clinically—whereas children in the waitlist condition did not. After the waitlist period was over, the control group also received the treatment program and showed a similar reduction in symptoms. For the full sample of 45 children, the effectiveness of the intervention was significant immediately after treatment and in 3- and 6-month follow-ups. In addition, children's anxiety cognition and their ability to cope with anxiety-provoking situations fully mediated the treatment gains. These results offer empirical support for cognitive-behavioral treatment programs in a nonWestern cultural context and plausible mediators for how cognitive-behavioral therapy works.
\end{abstract}

\title{
Key words:
}

Cognitive-behavioral therapy; Childhood anxiety; Efficacy; Effectiveness; Chinese; Group therapy 


\section{Effectiveness of Group Cognitive-Behavioral Treatment for Childhood Anxiety in Community Clinics}

From infancy onward, fear and anxiety can be adaptive because they heighten vigilance in threatening situations, but excessive fear and anxiety can cause distress. Anxiety disorders constitute the most commonly diagnosed psychological disorders for children and, if untreated, tend to persist over time (Anderson, 1994; Saavedra \& Silverman, 2002). Even when children recover from early anxiety disorders, they often develop others. Childhood anxiety disorders also predict problems in adulthood, including suicidal thoughts and attempts (Boden, Fergusson, \& Horwood, 2007) and an overall reduced quality of life (Olatunji, Cisler, \& Tolin, 2007). Even mild cases increase the risk for later anxiety, internalized symptoms, social incompetence, isolation, and shyness (Hirshfeld-Becker \& Biederman, 2002).

The Coping Cat program (Kendall, 1992), typically 15 to 20 sessions long, is arguably the best recognized and evaluated cognitive-behavioral therapy (CBT) protocol for childhood anxiety problems. Benefits have been found, both immediately and at 1-year follow-up, for American children with separation anxiety disorder, overanxious disorder, social phobia, and avoidant disorder (Kendall, 1994; Kendall et al., 1997). Adaptations of the program can be found in Canada (e.g., Coping Bear; Manassis, Avery, Butalia, \& Mendlowitz, 2004) and Australia (e.g., Coping Koala, Friends Program; Barrett, Dadds, \& Rapee, 1996).

The program's benefits have primarily been found in "efficacy" research-typically administered under optimal conditions in randomized controlled clinical trials with relatively homogeneous samples and minimal co-morbidities-leading to its classification as a "probably efficacious" treatment for both individuals and groups (APA Task Force, 1995; 
Flannery-Schroeder \& Kendall, 2000; Kendall, Hudson, Gosch, Flannery-Schroeder, \& Suveg, 2008; Manassis et al., 2002; Silverman et al., 1999). By contrast, "effectiveness" research assessing how well the program works under real-world conditions-with more heterogeneous clients, varying theoretical approaches and a range of clinical skills among therapists - has been quite rare (Southam-Gerow \& Kendall, 2000), so it remains unclear how effective CBT is for treating childhood anxiety. Both efficacy and effectiveness are crucial in treatment evaluation (Barkham \& Mellor-Clark, 2003). Importantly, there are widely accepted evaluation standards for both (Flay et al., 2005).

The present study has three main goals: (1) to evaluate the effectiveness of this "probably efficacious" treatment of childhood anxiety; (2) to examine its use in a nonWestern culture; and (3) to explore possible mediators of change.

This study is a response to an urgent need for more effectiveness evaluations. In a meta-analysis on childhood anxiety psychotherapy outcomes, none of the studies met the trio of criteria for effectiveness evaluation: clinically-referred and treatment-seeking participants, therapists with an active clinical practice, and a community clinical setting. Indeed, any one criterion was met in less than 5\% of the studies (Weisz, Doss, \& Hawley, 2005). As a result, little is known about the effectiveness of child psychotherapy (Cartwright-Hatton, Roberts, Chitsabesan, Fothergill, \& Harrington, 2004; Kendall \& Beidas, 2007). A randomized trial in outpatient settings found traditional non-CBT child psychotherapies to be no more effective than an individual-tutoring placebo (overall effect size $=-.08$; Weiss, Catron, Harris, \& Phung, 1999), despite their well-documented efficacy for anxiety-depression, aggression, and attention problems/hyperactivity. These findings may reflect the difficulty of adhering to treatment protocol in community clinics, given the many competing demands in real-world settings. 
More promising results emerged from two randomized trials comparing CBT and Treatment-as-Usual (TAU: child psychotherapy, family therapy, and eclectic treatments) in community mental health service. In perhaps the first published study of its kind, Barrington, Prior, Richardson, and Allen (2005) found that 7- to 14-year-olds with anxiety disorders treated with either CBT or TAU improved on a variety of measures (child, parent, teacher reports; clinical interviews by clinical psychologists blind to treatment conditions). From pretreatment baseline to follow-ups at 3, 6, and 12 months after treatment onset, the improvement was significant for both types of treatment, with no significant differences between the two. In another study conducted on a diverse sample of Caucasian, African American, and Latino/Latina 8- to 15-year-olds diagnosed with depressive disorders and multiple co-morbidities (Weisz et al., 2009), CBT and usual clinical care again did not differ significantly, but both effectively reduced depressive symptoms to sub-clinical levels posttreatment for $75 \%$ of the youths.

These two studies stand out among the many efficacy evaluations of CBT for childhood anxiety problems and reveal its promise as an effective treatment for childhood psychopathology. Yet because neither study directly compared CBT to a waitlist control, it remains unclear to what extent the observed improvement reflects treatment effectiveness versus spontaneous remission. The present study addresses this problem.

A second goal of this study is to evaluate the use of CBT with Chinese children. What we know about CBT efficacy thus far is based primarily on Caucasian middle-class samples, but evidence-based therapy originally developed with such a population may not transfer directly to other populations (e.g., Sue, Ivey, \& Pedersen, 1996). In particular, traditional, individual-oriented psychotherapy may not work quite as well for more collectivistic cultures (e.g., Hwang, Wood, Lin, \& Cheung, 2006; Wood, Chiu, Hwang, Jacobs, \& Ifekwunigwe, 2008). 
On the promising side of the ledger, there is evidence that adapting programs developed in one culture for use in another can be successful. A culturally-adapted group CBT program has been found to benefit Chinese adults with chronic depression (Wong, 2008). Moreover, in two case studies, with certain cultural modifications, CBT led to positive outcomes for children from more collectivistic cultural backgrounds (a MexicanAmerican child with separation anxiety, Wood et al., 2008; a Chinese-American child with school phobia, Hwang et al., 2006). Indeed, in general, interventions targeted at specific cultural groups seem much more beneficial than culturally generic ones (Griner \& Smith, 2006).

CBT stands a decent chance to be compatible with Chinese cultural norms. The structured counseling sessions have concrete goals and well-defined social roles (Lin \& Cheung, 1999). The education model of CBT relates well to the traditional Chinese belief that diligent learning brings about desirable changes (Chen \& Davenport, 2005; Hwang, 2006; Lin, 2002). Yet, Chinese children generally are not encouraged to discuss their emotions and feelings openly. For instance, American mothers and young children often talk about the causes of emotions that the children experience, such as happiness, sadness, fear, and anger ("emotion-explaining style"). By contrast, Chinese mothers tend to be more didactic—often criticizing the "perpetrators" who hurt other people's feelings and using such conversations to teach proper behavior ("emotion-criticizing style"; Wang, 2001). So, Chinese children may find it difficult to discuss their anxiety in a CBT session, especially in a group setting. Such reticence can make group CBT difficult. Moreover, the relative novelty of CBT group therapy for children in Hong Kong may also render parents more skeptical and hesitant about starting, and less committed to completing, their children's treatment program. It remains to be seen how well CBT works for Chinese children. 
A third goal—if CBT proves beneficial to Chinese children with anxiety problemsis to understand better the mechanism of change. We know reasonably well how cognition can lead to anxiety. Anxious individuals tend to overestimate danger (Beck, Emery, \& Greenberg, 1985) and underestimate their ability to control outcomes, thereby leading to negative physiological reactions and anxiety (Alloy, Kelly, Mineka, \& Clements, 1990; Barlow, 2002). Children's perceived control over threats predicts their self-reported anxiety (Weems, Silverman, Rapee, \& Pina, 2003). Anxious children underestimate their abilities to deal with danger (Bogels \& Zigterman, 2000) and overestimate physical and social threats (Schniering \& Rapee, 2004). Moreover, anxious children facing ambiguous situations tend to make more threat interpretations and avoidant-action plans (Barrett, Rapee, Dadds, \& Ryan, 1996; Chorpita, Albano, \& Barlow, 1996). But what is it about CBT treatment that changes these anxious perceptions and reactions? This study explores two candidate mediators of CBT treatment effects for childhood anxiety, namely, reducing anxious — as distinct from positive or depressed — self-statements (Kendall \& Treadwell, 2007), and improving coping behavior (Chu \& Harrison, 2007).

There are two main hypotheses in this study:

1. Culturally adapted CBT will be an effective as well as efficacious treatment for Chinese children with anxiety problems.

2. Reduction in anxiety cognition and improvement in coping behavior will significantly mediate the treatment effects.

\section{Method}

\section{Participants}

Forty-five Chinese children of Hong Kong origin (aged 6 - 11; mean $=8$ yrs 7 mos, $\mathrm{SD}=14$ mos) diagnosed with anxiety problems/disorders participated with parental consent. The children included 24 boys and 21 girls from primarily working and middle-class two- 
parent households (median household income around US\$2,500/month). They were referred by physicians or psychologists to the Child Assessment Service (a government agency in Hong Kong) for one or more of these concerns: learning (40\%), behavior (22\%), moodrelated (24\%), anxiety (13\%), other developmental (13\%) problems. In the Child Assessment Service intake interview, 28 parents (62\%) expressed concerns about learning or behavior, 22 (49\%) mentioned mood-related problems (e.g., social communication problem, temper tantrum, crying behavior, irritability), and only 4 (9\%) mentioned anxiety problems.

Among these 45 children, $38 \%$ were diagnosed with generalized anxiety disorder, $24 \%$ with separation anxiety disorder, and $51 \%$ with social phobia. Eight children (18\%) did not meet DSM-IV-TR criteria but had sub-clinical symptoms of anxiety disorders that interfered with daily functioning. (Children with only specific phobias were excluded. One child was excluded due to severe hyperactivity symptoms not managed by prescription medication.) Furthermore, $23 \%$ were co-morbid with dyslexia, 14\% with attention deficit/hyperactivity disorder, $7 \%$ with developmental coordination disorder, $16 \%$ with specific language impairment, and 7\% with selective mutism. All 45 children had normal IQ (within $1 \mathrm{SD}$ of the local norm on the Hong Kong Wechsler Intelligence Scale HK-WISC; Psychological Corporation, 1981). The learning disability diagnoses were based on standardized tests with local norms such as Hong Kong Test of Specific Learning Difficulties in Reading and Writing (using 1 SD below the mean on HKT-SpLD as clinical cutoff; Ho, Chan, Lee, Tsang, \& Luan, 2004), in addition to DSM-IV-TR. The observed co-morbidity prevalence was typical of local community clinics. All of the children were attending mainstream elementary schools. When probed, 32 parents $(71 \%)$ reported that their children had exhibited anxiety symptoms since kindergarten. 


\section{Procedure}

The study was conducted in one of the six government community clinics set up to help serve children with developmental challenges in Hong Kong. All of the clinical psychologists had a professional master's degree in clinical psychology (the local entry qualification), which included basic CBT training; some were working towards a professional doctorate in clinical child psychology. In general, physicians and psychologists refer children to this clinic because of developmental concerns. During the study, when any child was identified at the clinic as showing anxiety symptoms, the child and parent(s) were told about the study. If parents showed interest, a clinical psychologist on our research team then called them, explained the study, and obtained oral consent for a structured telephone interview about the referred child's anxiety symptoms. Eligible children (i.e., those with anxiety symptoms that interfered with everyday functioning) and their parents were then invited to join the study. After securing signed informed consent from parents, we asked the parents to provide demographic information. The children were then randomly assigned to either the treatment or waitlist-control condition. Figure 1 presents a CONSORT flow chart.

In the treatment condition, children were given a baseline assessment, received 8 weekly sessions of treatment, a $9^{\text {th }}$ and final treatment session after a 2 -week break, and then a post-treatment assessment within 2 weeks of completing the treatment. The time interval between the first and second assessment (Time $1 \&$ Time 2 ) was on average 13 weeks. In the waitlist-control condition, children were given two baseline assessments (Time $1 \&$ Time 2), also 13 weeks apart on average to serve as controls. The interval between assessments ranged from 12 to 14 weeks for both conditions. After their second assessment, children in the control group were given the same 9-session treatment that children in the treatment condition had received and then a post-treatment assessment (Time 3). Interviews and questionnaire measures were administered individually at these assessments to children and 
parents, who also completed questionnaires at 3-month and 6-month post-treatment followups.

Treatment Program. Our Anxiety Group-Treatment Program (Chan, 2006) was adapted with permission from Flannery-Schroeder and Kendall's (1996) Coping Cat CBT group treatment program by an experienced local clinical psychologist. The philosophy of CBT and the core elements of the original program were preserved. In line with Hwang's (2006) Psychotherapy Adaptation and Modification Framework, our adaptation focused on:

(1) Meeting the needs of Chinese children and parents. The treatment sessions were conducted in the children's and clinicians' native language, namely Cantonese Chinese. The acronym FEAR used in the Coping Cat program was replaced with an easy-to-remember Chinese acronym — explained to the children in simple language — to highlight core CBT elements: recognizing anxiety symptoms, combating cognitive bias with cognitive restructuring, practicing gradual exposure to anxiety-provoking stimuli, and evaluating and rewarding one's own coping.

The original sixteen 1-hour sessions in the Coping Cat program have been adapted into twelve 1.5-hour sessions in the Coping Bear program (e.g., Manassis et al., 2002). We further re-packaged the protocol into nine 2-hour sessions to make it more feasible in light of the hectic family schedules and limited mental-health care resources in Hong Kong. This change reduced travel time and overhead for each session (e.g., waiting for children to arrive and settle down). The children stayed engaged in the 2-hour sessions, thanks to puppet play, competitive games, worksheets, and the question-and-answer format (see also Kendall, Chu, Gifford, Hayes, \& Nauta, 1998). Moreover, having psycho-education and coaching scheduled in the first half of each session and exposure and relaxation/breathing exercises in the second half helped sustain even the youngest children's interest. 
To compensate for having fewer sessions, we maximized exposure practice by starting in-vivo exposure in the third session. Each group session consisted of 7 to 9 children who were further divided into three smaller groups for individualized exposure tasks. For example, children were asked to buy stationery from friendly as well as unfriendly store clerks (who were actually clinic staff not otherwise involved in this study). For homework, parents were encouraged to find real-world practice opportunities, and children were asked to complete worksheets with Chinese instructions at first-grade reading level. (Summary treatment protocol is available upon request.)

2) Strengthening the client-therapist relationship. Parents were invited to learn coaching techniques by observing treatment sessions and to meet with one of the CBT coleaders for about 20 minutes in the $1^{\text {st }}, 2^{\text {nd }}, 5^{\text {th }}, 8^{\text {th }}$, and $9^{\text {th }}$ sessions, thus facilitating communication. In particular, these sessions began with one clinical psychologist (assisted by a helper) working with the children in the therapy room and a second clinical psychologist briefing the parents in another room on the treatment and how they could help. Then the second clinical psychologist joined the treatment session in progress and invited the parents to observe in the back of the therapy room. In the parent-briefing at the $8^{\text {th }}$ treatment session, the concerns of both children and parents were discussed; at the $9^{\text {th }}$-session briefing, parents were motivated to ensure that real-world in vivo exposure practice would continue for their children. Parents were not required to attend all five beginning-of-session briefings because most parents work long hours in Hong Kong, and children took home session summaries with suggestions for how parents could help.

Anticipating that Chinese parents and children might be reticent, the clinicians invited questions before ending each session. Also, because absence in the early sessions might reflect unreported difficulties, the treatment program coordinator telephoned the parents of the few children who had missed the first or second session to reduce potential dropouts. 
(3) Taking into account Chinese notions of mental health and well-being. Our Chinese kung fu analogy encouraged children to learn and practice muscle relaxation, capitalizing on the cachet of kung fu for improving mental and physical health. Further, we capitalized on children's intuitive biological concept of vital force (akin to the Asian concept of "qi," or "breath/vital energy"; Hatano \& Inagaki, 1994) to teach children diaphragmatic breathing. Moreover, because anxiety often carries a social stigma among Chinese, clinic psychologists offered psycho-education on anxiety prior to treatment, presenting efficacy data on CBT and the professional qualifications of the treatment team. The treatment goals and the roles of therapists and participants were also explicitly set (see also Wong, 2008).

Program Staff \& Treatment Adherence. A team of 6 full-time staff clinical psychologists with on average 9 years of clinical experience $(\mathrm{SD}=5$ years, ranging from 4 to 18 years; all women) at the clinic ran the CBT sessions as part of their workload. They all had basic CBT training, and two (co-authors Chan and Lau) had further training on CBT group treatment for children (e.g., attending workshops and seminars given by experts such as R. Rapee and P. Barrett, visiting labs such as J. Wood's lab at UCLA). Chan (2006), who adapted the treatment protocol from Coping Cat, had also regularly conducted group CBT at the clinic. To ensure treatment adherence, Chan met with the other clinical psychologists beforehand to go over a set of treatment notes (Chan, 2006) and agree on specific CBT techniques. The 24 children in the treatment condition were divided into three groups, with two clinical psychologists (co-leaders) assigned to each group of 7 to 9 children. To mimic a team of one experienced and one less experienced therapist typical for this group size (e.g., Manassis et al., 2002), at least one co-leader had some expertise in CBT group treatment for children (either Chan or Lau). After the waitlist period, the 21 waitlist-control children were also divided into three CBT groups for treatment. 
Each treatment team also included either a clinic nurse or a clinical psychology intern to assist children with additional developmental challenges (e.g., dyslexia, attention deficits). To compensate for having fewer sessions, co-leaders assisted by the helper sometimes subdivided their groups into three smaller groups for more individualized exposure practice. The helpers were briefed on their role in such practice beforehand. Immediately after each session, both co-leaders independently recorded on a checklist whether the session protocol had been followed. Such records revealed excellent treatment adherence, with $100 \%$ of the required components implemented in every session.

\section{Structured Diagnostic Interview}

Kiddie-Schedule for Affective Disorders and Schizophrenia (K-SADS). This semistructured interview was used for diagnosing childhood psychopathology according to DSMIV-R (Kaufman, Birmaher, Brent, Rao, \& Ryan, 1997). A clinical psychologist conducted, audiotaped, and coded the telephone K-SADS parent interviews. A second clinical psychologist, who was not otherwise involved in this study, independently coded the audiotaped interviews of a random sample of $38 \%$ (17 out of 45$)$ of the children. To keep the second coder blind to whether a child was in the treatment or waitlist-control condition and whether an interview was pre- or post-treatment, the interviewer avoided any discussion of treatment status and assessment timing in the K-SADS interviews. For all four anxiety disorders (social anxiety, separation anxiety, generalized anxiety, specific phobias), the intercoder reliability was excellent as to whether individual children were within clinical range, sub-clinical range, or normal range (Kappas $>.92$ ). Test-retest reliability was assessed, for this and other measures, across the 13-week waitlist period in the control group. The retest stability was $100 \%$ for the K-SADS diagnoses (Kappas $=1.0)$.

\section{Child Self-Report Measures}


Treatment effect was evaluated with Spence's Children's Anxiety Scale (SCAS) and the Positive and Negative Affect Schedule (PANAS). Potential mediators - anxiety cognition and coping - were measured by the Negative Affectivity Self-Statement Questionnaire (NASSQ) and Coping Questionnaire (CQ-C) for children respectively.

Spence's Children's Anxiety Scale (SCAS). Good convergent and discriminant validity has been documented with Australian children for this self-report measure (Spence, 1998). To obtain a local Chinese reference sample, we asked $2071^{\text {st }}$ to $4^{\text {th }}$ grade children (103 boys and 104 girls, aged 6 to 11 years) in two mainstream elementary schools in Hong Kong to complete a Chinese version of SCAS translated by Wang and Deng (2004). The Cronbach's alpha was .94; the mean total for the four anxiety sub-scales (separation anxiety, social phobia, physical-injury anxiety, and generalized anxiety) was $23.9(\mathrm{SD}=13.0)$ and comparable to Spence's (1998) Australian normative sample (mean =21.7; SD =14.0). For our intervention study sample, Cronbach's alpha was .85 at Time 1 and .90 at Time 2. The retest reliability (Pearson $r)$ was $.76(p<.001)$.

Positive and Negative Affect Schedule (PANAS). Children rated how often they had experienced positive affect (e.g., interested, strong, proud, attentive; PANAS-PA) and negative affect (e.g., upset, scared, hostile, ashamed; PANAS-NA) in recent weeks (Watson, Clark, \& Tellegen, 1988; Laurent et al., 1999) to assess psychological well-being. This and other measures without a published Chinese version were translated and back-translated by professionals to ensure semantic equivalence. Cronbach's alpha was .84 at Time 1 and .70 at Time 2 for PANAS-PA, and .89 and .87 respectively for PANAS-NA. The retest reliability was .56 for positive affect $(p s<.01)$ and .74 for negative affect $(p<.001)$.

Children’s Negative Affectivity Self-Statement Questionnaire (NASSQ). Children indicated how often anxious thoughts occurred during the past week (e.g., "I am going to make a fool of myself'). Concurrent and construct validity, as well as internal and retest 
reliability, have been documented for this instrument (Ronan, Kendall, \& Rowe, 1994). In the present study, the 11-item scale for 7- to 10-year-olds was used; Cronbach's alpha was .65 at Time 1 and .66 at Time 2 . The retest reliability was $.58(p<.01)$.

Coping Questionnaire - Child (CQ-C). Children rated how well they coped with three anxiety-provoking situations identified in the K-SADS interview; its sensitivity to treatment effect has been documented (Kendall, 1994). Cronbach's alpha was modest with only three items (.47 at Time 1 and .57 at Time 2$)$. The retest reliability was only .26 but became .60 upon removing an outlier $(p<.01)$.

\section{Parent Report Measures}

Spence's Children's Anxiety Scale - Parent (PSCAS). This parent-report of child anxiety paralleled the SCAS (Spence, 1998). We asked the parents of the 207 children in our local reference sample to complete the PSCAS in Chinese (translated by Wang, 2005).

Cronbach's alpha was .92; the mean total of the four anxiety sub-scales was $20.5(\mathrm{SD}=9.4)$ and higher than the only published norms to date $($ mean $=14.3, \mathrm{SD}=11.6$, for a similar age range in Australia; Nauta et al., 2004). For our sample, Cronbach's alpha was .83 at Time 1 and .86 at Time 2 . The retest reliability was $.88(p<.001)$.

Coping Questionnaire - Parent (CQ-P). This parent-report of child anxiety-coping paralleled the CQ-C. Its sensitivity to treatment effect has been documented (Kendall, 1994). Cronbach's alpha in our study was .62 at Time 1 and .78 at Time 2 . The retest reliability was .64 $(p<.005)$.

\section{Results}

\section{Factor Structure of the Spence Children's Anxiety Scale}

To see how well the factor structure of SCAS and PSCAS align between the original English-speaking norming samples in Australia (Spence, 1998; Nauta et al., 2004) and our 
local Chinese reference sample, we conducted confirmatory factor analysis (CFA) using EQS (Bentler, 1995) and following Spence's and Nauta et al.'s procedures closely. We examined their Models 1 to 5 using these criteria: i) $\chi^{2}$ change between models, ii) goodness-of-fit indices, and iii) factor loadings of individual items onto corresponding factors. Table 1 presents fit indices for Models 3 to 5 (further details are available upon request).

Model 3, loading all SCAS/PSCAS symptoms onto six correlated factors, seemed satisfactory and fitted the data better, by $\chi^{2}$ change, than the more restrictive Model 1 (i.e., one factor) and Model 2 (i.e., six uncorrelated factors). For SCAS, the NFI, NNFI, and CFI were above .96 for our local data and above .93 for Spence's (1998). For PSCAS, these three indices for our local data were above .97 and RMSEA below .01, suggesting excellent fit, compared to around .90 and .08 respectively for Nauta et al.'s (2004).

Spence (1998) favored Model 4 (six correlated factors and one general higher-order factor) for SCAS, and Nauta et al. (2004) favored Model 5 (five correlated factors and one higher order factor) for PSCAS. Our local SCAS and PSCAS data fit both Models 4 and 5 well (the NFI, NNFI, and CFI were above .96), except Model 4 for SCAS seemed too good to be true - with all items loaded onto a factor (probably GAD) leaving no unexplained variance (Byrne, 2006). Factor loadings of individual SCAS items in our sample on factors corresponding to Spence's (1998) best-fitting Model 4 were all significant $(p s<.01)$, ranging from .32 to .78 with a mean of .59 , quite comparable to Spence's, ranging from .41 to .76 with a mean of .57. For PSCAS, loadings for individual items in our sample on factors corresponding to those in Nauta et al.'s best-fitting Model 5 (2004) were all significant ( $p$ s $<.01$ ), ranging from .29 to .70 with a mean of .54 , quite comparable to Nauta et al.'s (2004), ranging from .29 to .83 with a mean of .60 .

Thus, our confirmatory factor analyses revealed that the Chinese translations of SCAS and PSCAS have factor structures and individual-item loadings on corresponding factors 
comparable to the original English versions. We now turn to the main goal of this study, namely, evaluating group CBT intervention for Chinese children with anxiety concerns.

\section{Treatment versus Waitlist-Control Group at Baseline Assessment}

To see if the assignment of children to the treatment versus the control condition was truly random, demographic information was compared between these two groups using $t$-tests and chi-square analyses. One child dropped out from the treatment condition, and three dropped out from the waitlist-control condition (Figure 1). Twenty-four children remained in the treatment condition (mean age $=8$ yrs 4 mos, $\mathrm{SD}=16$ mos; 11 boys, 13 girls), and 21 in the waitlist control (mean age $=8$ yrs 10 mos, $\mathrm{SD}=14$ mos; 13 boys, 8 girls). The two groups did not differ significantly by gender composition, $\chi^{2}(1, n=45)=.28$, n.s., or age, $t(43)$ $=1.42$, n.s. Other demographic characteristics (e.g., family income, percentage of singleparent households, parents' age) likewise did not differ significantly between the two conditions (all ps >.2).

Children in the two conditions did not differ significantly on the number of anxiety disorders (e.g., social phobia, separation anxiety disorder, generalized anxiety disorder), or prevalence of co-morbidity with ADHD, developmental coordination disorder, specific language impairments, and selective mutism (all ps $>.3$ ). Co-morbidity with dyslexia, however, was higher in the waitlist-control than in the treatment condition (38\% vs. $4 \%$ respectively, $p<.01)$. Importantly, child- and parent-reported anxiety levels did not differ significantly between the two conditions at Time 1 assessment $(F \mathrm{~s}(1,43)<2.9, p \mathrm{~s}>.05$; see Table 2 for means and standard deviations).

\section{Treatment Effects: Change from Time 1 to Time 2 Assessment}

To evaluate the effectiveness of the CBT group treatment, we chose the more conservative intent-to-treat analysis rather than treatment-completer analysis. All 24 children in the treatment condition were included in the analysis, even though some did not attend all 
nine treatment sessions. Preliminary ANOVAs revealed no significant 3-way interactions among Time (Time 1 vs. Time 2), Condition (Treatment vs. Waitlist), and any of these variables: gender, age, co-morbidity of anxiety disorders and other developmental disorders. Treatment effects were then evaluated by Condition $\mathrm{x}$ Time repeated-measure ANCOVAs (Table 2), with age, gender and dyslexia status included as covariates.

\section{Child Self-Report of Anxiety Level}

A repeated-measure ANCOVA on SCAS revealed a significant Condition x Time interaction, $F(1,40)=14.5, p<.001$. The average self-reported anxiety level decreased by 9.7 in the treatment group but increased by 1.8 in the waitlist-control group from Time 1 to Time 2.

\section{Parent Report of Child Anxiety}

An ANCOVA on PSCAS revealed a significant analogous interaction, $F(1,40)=4.7$, $p<.05$. The average parent-reported child anxiety level decreased by 4.2 in the treatment group but increased by 1.3 in the waitlist-control group from Time 1 to Time 2 .

\section{Child Self-Report of Global Affect}

An analogous ANCOVA on PANAS revealed no significant Condition $\mathrm{x}$ Time interaction for either positive affect (PANAS-PA; $F(1,40)=3.7, p=.06$ ) or negative affect $($ PANAS-NA; $F(1,40)=1.2, p=.28)$.

\section{Potential Mediators}

We also measured two potential mediators: children's anxiety cognition (NASSQ) and coping with anxiety-provoking situations (child-report CQ-C and parent-report CQ-P). Repeated-measure ANCOVAs revealed a significant Condition x Time interaction for each measure, $F \mathrm{~s}(1,40)>5.1$, $p \mathrm{~s}<.05$. The pattern of group means revealed a substantial reduction in self-statements about anxiety cognition and substantial improvement in anxiety coping from Time 1 to Time 2 in the treatment condition but not in the waitlist-control 
condition (Table 2). Anxiety cognition and the ability to cope with anxiety-provoking situations, then, are potential mediators for the observed CBT treatment effects for childhood anxiety. This mediation hypothesis will be evaluated presently, after a more in-depth look at the treatment effects.

\section{Analysis of Clinical Significance}

Following Kendall, Marrs-Garcia, Nath, and Sheldrick (1999), we examined clinical significance. First, we performed a clinical equivalency test. The mean SCAS score at Time 2 for the treatment group was 24.6, which was within one standard deviation of Spence's (1998) normative sample mean $(21.7 ; \mathrm{SD}=14)$. The clinical equivalency test indicated that the treated group returned to the range of the normative population (C.E. $t(2079)=4.2, p$ $<.001)$. We then performed a traditional $t$-test to compare the post-treatment group and Spence's normative group, which revealed no significant group difference $(\operatorname{Trad} t(2079)=.7$, n.s.). Together, these two tests demonstrated the clinical significance of the treatment effect.

Post-treatment SCAS of children in the treatment group was also compared to our local reference data. The clinical equivalence test and traditional $t$-test revealed that the treatment effect met both criteria of clinical significance (C.E. $t(229)=4.7, p<.001$; Trad $t$ $(229)=-.3$, n.s.). This means that the post-treatment self-reported anxiety level of the CBT group had returned to within the normal range, with reference to both an overseas normative sample and a local one. Importantly, no analogous return to the normal range of mean SCAS score was observed in the waitlist-control condition (mean $=38.8, \mathrm{SD}=13.7$ at Time 2$)$.

An analogous evaluation of clinical significance was performed on PSCAS. Nauta et al.'s (2004) normative sample yielded a mean score $14.3(\mathrm{SD}=11.6)$ for parents with children aged 6 to 11 in Australia. The mean PSCAS score for our local normative sample (i.e., parents of children age 6 to 11 in Hong Kong) was 20.5 (SD =9.4). The post-treatment PSCAS mean score in our treatment condition $(=28.8)$, although significantly lower than that 
in the control condition $(=36.5)$, was nonetheless significantly higher than that of Nauta et al.'s normative sample and our local one (by Trad t-tests, $p \mathrm{~s}<.05$ ). According to the parents, then, the anxiety level of children in our treatment condition did not return to normal functioning after treatment.

\section{Diagnostic Status}

According to K-SADS interviews, no child in the control condition showed any change in diagnosis status (i.e., 17 with anxiety disorders and 4 with sub-clinical anxiety problems) after the 13-week waitlist period. By contrast, in the treatment group, 13 of the 20 children $(65 \%)$ who had met DSM-IVR criteria for anxiety disorder before treatment no longer did so after treatment. Three of the 4 children (75\%) with anxiety symptoms in the sub-clinical range before treatment moved to the normal range afterwards. The treatment group and waitlist group differed significantly $\left(\chi^{2}(2, \mathrm{n}=45)=12.5, p<.01\right)$.

Recall that children in the waitlist-control condition also received treatment after the second baseline assessment. Because children in the treatment condition and control condition did not differ significantly on anxiety measures at the Time 1 baseline, they could be pooled together to evaluate treatment effects. We compared the pre- and post-treatment diagnostic status (Time 1 and Time 2 for the treatment condition; Time 2 and Time 3 for the waitlist-control) of the full sample of 45 children and found that $71 \%$ improved after treatment (Figure 2). Specifically, 14 children who began in the clinical range improved to having only sub-clinical problems (31\%); 12 who began in the clinical range and 6 in the subclinical range moved to the normal range (27\% and $13 \%$ respectively).

Treatment-completer analysis revealed a similar picture. Four children dropped out after one to three treatment sessions. (Two in sub-clinical range- one per conditiondropped out because their anxiety problems were not considered by their parents to be serious. Two other children — both in clinical range and in the waitlist control- had cried and refused 
to participate in the treatment.) Among the 41 treatment completers, $74.3 \%$ of the 35 children who had met the diagnostic criteria for anxiety disorder before treatment no longer did so after treatment. All of the 6 children who initially had anxiety symptoms in the subclinical range moved to the normal range. Evidently, the CBT treatment effects are quite compelling, yet an important question remains: Do such treatment effects persist over time?

\section{Maintenance of Treatment Effect}

Follow-ups at 3 months and 6 months post treatment were conducted on all 45 children. Repeated-measure ANOVAs across four assessments (pre-treatment, posttreatment, 3-month follow-up, 6-month follow up) revealed significant differences across assessments for self- and parent-report of child anxiety (SCAS, PSCAS), children's selfstatement of anxiety cognition (NASSQ), and children's ability to cope with anxietyprovoking situations (child-report $\mathrm{CQ}-\mathrm{C}$ and parent-report $\mathrm{CQ}-\mathrm{P}$ ), all $F \mathrm{~s}>7.1, p \mathrm{~s}<.01$, partial $E t a^{2} \mathrm{~s}>.36$. Figure 3 shows that much of the improvement took place from pretreatment to immediate post-treatment assessment, with the treatment effects holding steady in the 3-month and 6-month follow-ups. Post-hoc pairwise comparisons confirmed that pretreatment scores were significantly worse than those for immediate post-treatment, 3-month follow-up, and 6-month follow-up (all ts $>3.5$, bonferroni corrected $p \mathrm{~s}<.01$ ). The three post-treatment assessments did not differ significantly. The treatment effects persisted quite well over time and held steady even 6 months after treatment.

\section{Tests of Mediation}

To explore possible mediators of change, anxious thoughts (NASSQ) and constructive coping (CQ-C and CQ-P) were examined with the multiple mediation model (e.g., Cheung, 2007; MacKinnon, 2000; Preacher \& Hayes, 2008; Figures 4 and 5). The total effect of treatment status was significant: $B=-14.2, t=-3.9, p<.001$ for SCAS; and $B=-7.7, t=-2.4$, $p<.05$ for PSCAS. The total indirect effect of the three simultaneous mediators was also 
significant (for SCAS, $B=-10.8, z=-3.4, p<.001$; for PSCAS, $B=-6.6, z=-2.8, p<.01$ ), and the direct effect became non-significant (for SCAS, $B=-3.4, t=-1.3$, n.s., and PSCAS, $B$ $=-1.1, t=-.33$, n.s.). Together, NASSQ, CQ-C, and CQ-P appear to mediate the association between treatment status and both treatment outcomes (i.e., SCAS and PSCAS) completely.

Testing the specific contribution of each indirect effect (using normality-free bootstrap estimates) revealed that NASSQ and CQ-C significantly mediated the treatment status and the treatment outcome SCAS (-5.4 and -5.3, respectively), but CQ-P did not (-.11). For PSCAS, CQ-C significantly mediated the treatment status and outcome (-4.2), but NASSQ and CP-P did not (-1.9 and -.54 respectively).

\section{Discussion}

This study set out to narrow the gap between clinical research and clinical practice. The CBT group treatment program, which has been tested in well-controlled research settings, now reveals its usefulness for real-life patients treated by real-life therapists in a real-life clinic. The reduction in children's anxiety symptoms in the treatment condition, together with the absence of improvement in the control condition during the waitlist period, provides experimental evidence for CBT treatment benefits. Importantly, these findings held up well for both children's self-reports and parent-reports, and both clinical and statistical significance of treatment outcomes were documented. Indeed, children's self-reported anxiety returned to normative levels after treatment, according to both local and overseas reference samples. Follow-up at 3 months and 6 months after treatment suggested that the treatment effects were maintained well for at least 6 months. This randomized waitlistcontrolled study, then, replicated prior demonstrations of CBT efficacy for childhood anxiety.

More importantly, this study also demonstrated CBT effectiveness for clinically referred clients and treatment offered by clinic staff as part of their everyday caseload, in a publicly-funded community clinic with all the usual resource constraints and demands that 
clinical staff face in such settings (e.g., pressure to shorten long waitlists for clients, supervision of clinical psychology interns). Two prior studies suggested that group CBT was as effective as Treatment-as-Usual for childhood anxiety and depressive disorders (Barrington et al., 2005; Weisz et al., 2009). Yet because neither study directly compared CBT to a waitlist control, it was unclear to what extent the observed improvement reflected treatment effectiveness versus spontaneous remission. The present study documented significantly greater improvement in the CBT condition than in the waitlist control, thereby contributing much needed empirical evidence for the effectiveness of CBT group treatment for childhood anxiety and, more generally, for childhood psychotherapy (e.g., Weiss et al., 1999; Weisz et al., 2005, 2009; Epp \& Dobson, 2010).

Caution, however, is needed when interpreting these findings. We are mindful that the clinical psychologists could have biased parents in the structured interviews (K-SADS) or given biased diagnoses. However, the interviewer was an experienced full-time clinical psychologist at the clinic; she was conscientious in following the structured interview protocol, as a review of the audiotapes confirmed. Moreover, as noted earlier, another experienced clinical psychologist blind to the treatment status (treatment vs. waitlist) and timing of assessment (pre- vs. post-treatment) established excellent inter-coder reliability on anxiety diagnosis. Thus, bias in clinical assessment should not be a real concern for this study.

A second concern is that, although we worked hard to ensure that the Chinese translations of the measures were faithful to the English originals, the psychometrics for Chinese samples was relatively unknown. We therefore collected local reference data on Chinese children and their parents for two key measures, the Spence Children's Anxiety Scale child- and parent-reports (SCAS and PSCAS). The factor structures and loadings of individual items on corresponding factors were found to be comparable between our local 
community sample for the Chinese translated scales and English-speaking normative samples for the original scales published by Spence (1998) and Nauta et al. (2004).

Another potential concern is that we saw no spontaneous remission in the control group, whereas such remission has been observed in prior studies. This may be explained by how short the waitlist period was (only about 13 weeks) and how long the children's anxiety problems had persisted. Most children in this study began exhibiting anxiety symptoms in kindergarten, but their parents did not seek help until the children were in elementary school. Such delay in seeking treatment has been documented for Asian Americans (e.g., Sue, Fujino, Hu, Takeuchi, \& Zane, 1991). One reason may be scarcity of affordable mental health services. Another may be that Asian parents often underestimate the severity and impact of their children's anxiety problems. They may also delay seeking help because mental illnesses are seen by some Chinese as karmic retribution for ancestral or parental bad behaviors. The fear of social stigma may have also contributed to treatment avoidance. Perhaps we saw no remission in the control group because the children's anxiety problems were longstanding and hence relatively unlikely to go away spontaneously, especially during such a brief period.

A final concern is how well this relatively novel treatment will transport to other clinical settings in Hong Kong or elsewhere in Asia. Although the government clinic that housed this study is similar to other local community clinics in terms of heavy caseload, its staff is relatively well-remunerated and hence generally above average in experience and clinical skills. It remains to be seen if, and how much, additional staff training might be needed in other clinics to ensure the transportability of this CBT group treatment for children to other settings in Hong Kong and beyond.

Caveats aside, this study contributes much needed empirical evidence for the effectiveness of using group CBT to treat childhood anxiety. Moreover, this may well be the first ever such demonstration in a non-Western cultural context. The traditional Western 
individualistic approach has been considered inappropriate for counseling Chinese children growing up in a more collectivist culture. Nonetheless, the CBT group treatment turned out to work quite well (see also Wong, 2008). The low attrition rate and robust treatment effect of our adaptation of the Coping Cat Program could be attributed to our attention to cultural factors.

We tried to help parents and children feel at ease with this relatively novel treatment through psycho-education, using everyday examples and analogies based on Chinese cultural practices (e.g., kung fu, "qi," or breath/vital energy) to convey key concepts, and we worked to mitigate the reticence of Chinese children and parents about mental health concerns. Also, aware that the children had busy schedules and all of the parents were likely to have full-time jobs, we made the treatment program as convenient as possible by condensing it from sixteen 1-hour sessions to nine 2-hour sessions. Parents were invited to participate as collaborators, and children took home session summaries containing suggestions for how their parents could coach them outside the treatment group meetings. While parental involvement in our treatment program was not as intensive as some other programs (e.g., Manassis et al., 2002, with twelve concurrent child- and parent-group sessions), the encouragement and opportunities for parental involvement in our program may have nonetheless contributed to the effectiveness of this treatment program for Chinese children.

The children and parents responded well to our adaption of Coping Cat. The children readily accepted the psycho-education, especially the somatic education. Both children and parents liked the diaphragmatic breathing exercise. Given Chinese parents' preference for emotion-criticizing style over emotion-explaining style (Wang, 2001), we expected that many children — especially the youngest ones — would initially have difficulty identifying and talking about their automatic anxiety-inducing thoughts, and they did. But with our modeling of "emotion and feeling" talk and use of "thought bubble" cartoons, most children became 
better at identifying such thoughts. The beginning-of-session meetings with parents and endof-session questions-and-answers time elicited sharing from even initially reticent parents and over time generated more open discussion about their children's emotions and feelings.

Other findings are also of clinical interest. First, the effectiveness of the CBT group treatment was comparable across gender and the age range of 6 to 11 years, and across a range of co-morbid anxiety disorders and other developmental disorders. These findings support a more liberal application of CBT to children with anxiety disorders or problems.

Second, although all children in this study were clinically referred, few were referred by physicians specifically for anxiety. Only a few parents indicated anxiety as their major concern in the intake interview at the clinic. While some parents recognized that their children had behavioral or emotion-related problems (e.g., irritability, temper tantrums, crying, and/or having social communication difficulty), few saw these as anxiety symptoms. This may indicate that awareness of symptoms of childhood anxiety is inadequate among professionals (e.g., physicians) and parents alike. More public psycho-education seems in order.

Beyond evaluating the effectiveness of CBT group treatment for childhood anxiety, this study also speaks to possible mediators of change. Anxiety cognition (measured by child-reported NASSQ) and ability to cope with anxiety-provoking situations (measured by child-reported CQ-C) seem to significantly mediate the treatment effect on children's anxiety, as reported by parents as well as children (i.e., PSCAS and SCAS). These findings are in line with the outcomes of a meta-analysis of randomized clinical trials of CBT for child and adolescent anxiety (Chu \& Harrison, 2007), which found not only positive treatment gains but also a moderate effect size for positive change in cognitive and coping processes (see also Kendall \& Treadwell, 2007). Note, however, that temporal precedence of the mediators has 
not been established in this study. It remains to be seen whether these potential mediators are actual causal agents or simply covariates of change (Laurenceau, Hayes \& Feldman, 2007). Limitations notwithstanding, this randomized waitlist-controlled study is a rarity in the research literature - using practicing clinicians to treat clinically-referred children in a community clinical service setting (e.g., Weisz et al., 2005, 2009). Not only does it show that a CBT group treatment program can have a clinically significant impact in reducing anxiety symptoms in children, but it also helps narrow the gap between clinical research and clinical practice by demonstrating that empirically supported efficacious treatment can be effectively implemented in real-world clinics. 


\section{References}

Alloy, L. B., Kelly, K. A., Mineka, S., \& Clements, C. M. (1990). Comorbidity of anxiety and depressive disorders: A helplessness-hopelessness perspective. In J. D. Maser \& C. R. Cloninger (Eds.), Comorbidity of Mood and Anxiety Disorders (pp. 499-543). Washington, DC: American Psychiatric Press.

Anderson, J. C. (1994). Epidemiological issues. In T. H. Ollendick, N. J. King, \& W. Yule (Eds.), International handbook of phobic and anxiety disorders in children and adolescents: Issues in clinical child psychology (pp. 43-65). New York: Plenum Press. APA Task Force on Promotion and Dissemination of Psychological Procedures. (1995). Training in and dissemination of empirically validated psychological treatments: Report and recommendations. The Clinical Psychologist, 48, 3-24.

Barkham, M., \& Mellor-Clark, J. (2003). Bridging evidence-based practice and practicebased evidence: Developing a rigorous and relevant knowledge for the psychological therapies. Clinical Psychology and Psychotherapy, 10, 319-327. doi:10.1002/cpp.379

Barlow, D. H. (2002). Anxiety and its Disorders: The Nature and Treatment of Anxiety and Panic (2 ${ }^{\text {nd }}$ ed.). New York: Guilford Press.

Barrett, P. M., Dadds, S. M., \& Rapee, R. M. (1996). Family treatment of childhood anxiety: A controlled trial. Journal of Consulting and Clinical Psychology, 64, 333-342. doi:10.1037/0022-006X.64.2.333

Barrett, P. M., Rapee, R. M., Dadds, S. M. \& Ryan, S. M. (1996). Family enhancement of cognitive style on anxious and aggressive children. Journal of Abnormal Child Psychology, 24, 187-203. doi:10.1007/BF01441484

Barrington, J., Prior, M., Richardson, M., \& Allen, K. (2005). Effectiveness of CBT Versus Standard Treatment for Childhood Anxiety Disorders in a Community Clinic Setting. Behaviour Change. 22, 29-43. doi:10.1375/bech.22.1.29.66786 
Beck, A. T., Emery, G., \& Greenberg, R. (1985). Anxiety disorders and phobias: A cognitive perspective. New York: Basic Books.

Bentler, P. M. (1995). EQS Structural equations program manual. Encino, CA: Multivariate Software.

Boden, J. M., Fergusson, D. M. \& Horwood, L. J. (2007). Anxiety disorders and suicidal behaviours in adolescence and young adulthood: Findings from a longitudinal study. Psychological Medicine, 37, 431-440. doi: 10.1017/S0033291706009147

Bogels, S. M. \& Zigterman, D. (2000). Dysfunctional cognitions in children with social phobia, separation anxiety disorder, and, generalised anxiety disorder. Journal of Abnormal Child Psychology, 28, 205-211. doi: 10.1023/A:1005179032470

Byrne, B. M. (2006). Structural equation modeling with EQS: Basic concepts, applications, and programming. Mahwah, NJ: Lawrence Erlbaum.

Cartwright-Hatton, S., Roberts, C., Chitsabesan, P., Fothergill, C., \& Harrington, R. (2004). Systematic review of the efficacy of cognitive behaviour therapies for childhood and adolescent anxiety disorders. British Journal of Clinical Psychology, 43, 421-436. doi:10.1348/0144665042388928

Chan, C. K. (2006). The Anxiety Group-Treatment Program. Unpublished manuscript. Hong Kong: Child Assessment Service, Department of Health.

Chen, S. W. H., \& Davenport, D.S. (2005). Cognitive-behavioral therapy with Chinese American clients: Cautions and modifications. Psychotherapy: Therapy, Research, Practice, Training, 42, 101-110. doi:10.1037/0033-3204.42.1.101

Cheung, M. W.-L. (2007). Comparison of approaches to constructing confidence intervals for mediating effects using structural equation models. Structural Equation Modeling, 14, $227-246$. 
Chorpita, B. F., Albano, A. M., \& Barlow, D. H. (1996). Cognitive processing children: Relation to anxiety and family influences. Journal of Clinical Child Psychology, 25, 170-176. doi:10.1207/s15374424jccp2502_5

Chu, B.C., \& Harrison, T. L. (2007). Disorder-specific effects of CBT for anxious and depressed youth: A meta-analysis of candidate mediators of change. Clinical Child and Family Psychology Review, 10, 352-372. doi:10.1007/s10567-007-0028-2

Epp, A. M., \& Dobson, K. S. (2010). The evidence base for cognitive-behavioral therapy. In K. S. Dobson (Ed.), Handbook of cognitive-behavioral therapies ( $3^{\text {rd }}$ ed.; pp. 39-73). New York: Guilford Press.

Flannery-Schroeder, E., \& Kendall, P. C. (1996). Cognitive-behavior therapy for anxious children: Therapist manual for group treatment. Ardmore: Workbook Publishing. Flannery-Schroeder, E. C., \& Kendall, P. C. (2000). Group and individual cognitivebehavioral treatments for youth with anxiety disorders: A randomized clinical trial. Cognitive Therapy and Research, 24, 251-278. doi:10.1007/s10608-005-3168-z

Flay, B. R., Biglan, A., Boruch, R. F., Castro, F. G., Gottfredson, D., Kellam, S., Moscicki, E. K., Schinke, S., Valentine, J. C., \& Ji, P. (2005). Standards of evidence: Criteria for Efficacy, Effectiveness and Dissemination. Prevention Science, 6, 151-175. doi:10.1007/s11121-005-5553-y

Griner, D., \& Smith, T. B. (2006). Culturally adapted mental health intervention: A metaanalytic review. Psychotherapy: Theory, Research, Practice, Training, 43, 531-548. doi:10.1037/0033-3204.43.4.531

Hatano, G. \& Inagaki, K. (1994). Young children's naive theory of biology. Cognition, 50, 171-188. doi:10.1016/0010-0277(94)90027-2 
Hirshfeld-Becker, D. R. \& Biederman, J. (2002). Rationale and principles for early intervention with young children at risk for anxiety disorders. Clinical Child and Family Psychology Review, 5, 161-172.

Ho, C. S., Chan, D. W., Lee, S., Tsang, S., \& Luan, V. H. (2004). Cognitive profiling and preliminary subtyping in Chinese developmental dyslexia. Cognition, 91, 43-75. doi:10.1016/S0010-0277(03)00163-X

Hwang, W. C. (2006). The psychotherapy adaptation and modification framework: Application to Asian Americans. American Psychologist, 62, 702-715. doi:10.1037/0003-066X.61.7.702

Hwang, W. C., Wood, J. J., Lin, K. M., \& Cheung, F. (2006). Cognitive-behavioral therapy with Chinese Americans: Research, theory, and clinical practice. Cognitive Behavioral Practice, 13, 293-303. doi:10.1016/j.cbpra.2006.04.010

Kaufman, J., Birmaher, B, Brent, D. A., Rao, U., \& Ryan, N. D. (1997). Schedule for affective disorders and schizophrenia for school age children, present and lifetime version (K-SADS-PL): Initial reliability and validity data. Journal of the American Academy of Child and Adolescent Psychiatry, 36, 980-988. doi:10.1097/00004583199707000-00021

Kendall, P. C. (1992). Coping Cat Workbook. Ardmore, PW: Workbook Publishing. Kendall, P. C. (1994). Treating anxiety disorders in children: Results of a randomized clinical trial. Journal of Consulting and Clinical Psychology, 62, 100-110. doi:10.1037/0022-006X.62.1.100

Kendall, P. C., \& Beidas, R. S. (2007). Smoothing the trail dissemination of evidence-based practices for youth and flexibility within fidelity. Professional Psychology: Research and Practice, 38, 13-20. doi:10.1037/0735-7028.38.1.13 
Kendall, P. C., Chu, B., Gifford, A., Hayes, C., Nauta, M. (1998). Breathing life into a manual: Flexibility and creativity with manual-based treatments. Cognitive and Behavioral Practice, 5, 177-198. doi:10.1016/S1077-7229(98)80004-7

Kendall, P. C., Flannery-Schroeder, E., Panichelli-Mindel, S. M., Sourtham-Gerow, M., Henin A., \& Warman, M. (1997). Therapy for youths with anxiety disorders: a second randomized clinical trial. Journal of Consulting and Clinical Psychology, 65, 366-380. doi:10.1037/0022-006X.65.3.366

Kendall, P. C., Hudson, J.L., Gosch, E., Flannery-Schroeder, E., \& Suveg, C. (2008). Cognitive-behavioral therapy for anxiety disordered youth: A randomized clinical trial evaluating child and family modalities. Journal of Consulting and Clinical Psychology, 76, 282-297. doi:10.1037/0022-006X.76.2.282

Kendall, P. C., Marrs-Garcia, A., Nath, S. R., \& Sheldrick, R. C. (1999). Normative comparisons for the evaluation of clinical significance. Journal of Consulting and Clinical Psychology, 67, 285-299. doi:10.1037/0022-006X.67.3.285

Kendall, P. C., \& Treadwell, K. R. H. (2007). The role of self-statements as a mediator in treatment for youth with anxiety disorders. Journal of Consulting and Clinical Psychology, 75, 380-389. doi:10.1037/0022-006X.75.3.380

Laurenceau, J.-P., Hayes, A. M., \& Feldman, G. C. (2007). Some methodological and statistical issues in the study of change processes in psychotherapy. Clinical Psychology Review, 27, 682-695. doi:10.1016/j.cpr.2007.01.007

Laurent, J., Catanzaro, S. J., Joiner, T. E., Rudolph, K. D., Potter, K. I., Lambert, S., Osborne, L., \& Gathright, T. (1999). A measure of positive and negative affect for children: Scale development and preliminary validation. Psychological Assessment, 11, 326-338. doi:10.1037/1040-3590.11.3.326 
Lin, K.-M., \& Cheung, F. (1999). Mental health issues for Asian Americans. Psychiatric Services, 50, 774-780.

Lin, Y. N. (2002). The application of cognitive-behavioral therapy to counselling Chinese. American Journal of Psychotherapy, 56, 46-58.

MacKinnon, D. P. (2000). Contrasts in multiple mediator models. In J. Rose, L. Chassin, C. C. Presson, \& S. J. Sherman (Eds.), Multivariate applications in substance use research: new methods for new questions (pp. 141 - 160). Mahwah, NJ: Erlbaum.

Manassis, K., Avery, D., Butalia, S., \& Mendlowitz, S. (2004). Cognitive-Behavioral Therapy With Childhood Anxiety Disorders: Functioning in Adolescence. Depression and Anxiety, 19, 209-216. doi:10.1002/da.10133

Manassis, K., Mendlowitz, S. L., Scapillato, D., Avery, D., Fiksenbaum, L., Freire, M., Monga, S., \& Owens, M. (2002). Group and individual cognitive-behavioral therapy for childhood anxiety disorders: A randomized trial. Journal of the American Academy Child and Adolescent Psychiatry, 41, 1423-1430. doi:10.1097/00004583-20021200000013

Nauta, M. H., Scholing, A., Rapee, R. M., Abbott, M., Spence, S. H., \& Waters, A. (2004). A parent-report measure of children's anxiety; Psychometric properties and comparison with child-report in a clinic and normal sample. Behaviour Research and Therapy, 42, 813-839. doi:10.1016/S0005-7967(03)00200-6

Olatunji, B. O., Cisler, J. M., \& Tolin, D. F. (2007). Quality of life in the anxiety disorders: A meta-analytic review. Clinical Psychology Review, 27, 572-581. doi:10.1016/j.cpr.2007.01.015

Preacher, K. J., \& Hayes, A. F. (2008). Asymptotic and resampling strategies for assessing and comparing indirect effects in multiple mediator models. Behavior Research Methods, 40, 879-891. doi:10.3758/BRM.40.3.879 
Psychological Corporation. (1981). Hong Kong Wechsler Intelligence Scale for Children (HK-WISC) manual. New York: Psychological Corporation.

Ronan, K., Kendall, P. C., \& Rowe, M. (1994). Negative affectivity in children:

Development and validation of a self-statement questionnaire, Cognitive Therapy and Research, 18, 509-528. doi:10.1007/BF02355666

Saavedra, L. M., \& Silverman, W. K. (2002). Classification of anxiety disorders in children: What a difference two decades make. International Review of Psychiatry, 14, 87-100. doi:10.1080/09540260220132617

Schniering, C. A., \& Rapee, R. M. (2004). The structure of negative self-statements in children and adolescents: A confirmatory factor-analytic approach. Journal of Abnormal Child Psychology, 32, 95-109. doi:10.1023/B:JACP.0000007583.90038.7a

Silverman, W. K., Kurtines, W. M., Ginsburg, G. S., Weems, C. F., Lumpkin, P. W., \& Carmichael, D. H. (1999). Treating anxiety disorders in children with group cognitivebehavioral therapy: A randomized clinical trial. Journal of Consulting and Clinical Psychology, 67, 995-1003. doi:10.1037/0022-006X.67.6.995

Southam-Gerow, M. A., \& Kendall, P. C., (2000). Cognitive-behaviour therapy with youth: Advances, challenges, and future directions. Clinical Psychology and Psychotherapy, 9, 343-366. doi:10.1002/1099-0879(200011)7:5<343::AID-CPP244>3.3.CO;2-0

Spence, S. H. (1998). A measure of anxiety symptoms among children. Behaviour Research and Therapy, 36, 545-566. doi:10.1016/S0005-7967(98)00034-5

Sue, S., Fujino, D. C., Hu, L., Takeuchi, D. T., \& Zane, N. W. S. (1991). Community mental health services for ethnic minority groups: A test of the cultural responsiveness hypothesis. Journal of Consulting and Clinical Psychology, 59, 533-540. doi:10.1037/0022-006X.59.4.533 
Sue, D. W., Ivey, A. E., \& Pedersen, P. V. (1996). A theory of multicultural counseling and therapy. Belmont, CA: Brooks/Cole Publishing Co.

Wang, Q. (2001). "Did you have fun?" American and Chinese mother-child conversations about shared emotional experiences. Cognitive Development, 16, 693-715. doi:10.1016/S0885-2014(01)00055-7

Wang, W. (2005) Chinese version of the Spence Children's Anxiety Scale—parent version. http://www.scaswebsite.com/docs/scas-parent-chinese.pdf

Wang, W., \& Deng, C. (2004). Chinese version of the Spence Children's Anxiety Scalechildren version. http://scaswebsite.com/docs/scas-child-chinese.pdf

Watson, D., Clark, L. A., \& Tellegen, A. (1988). Development and validation of brief measures of Positive and Negative Affect: The PANAS scales. Journal of Personality and Social Psychology, 54, 1063-1070. doi:10.1037/0022-3514.54.6.1063

Weems, C. F., Silverman, W. K., Rapee, R., \& Pina, A. A. (2003). The role of control in childhood anxiety disorders. Cognitive Therapy and Research, 27, 557-568.

Weiss, B., Catron, T., Harris, V., \& Phung, T. M. (1999). The effectiveness of traditional child psychotherapy. Journal of Consulting and Clinical Psychology, 67, 82-94. doi:10.1037/0022-006X.67.1.82

Weisz, J. R., Doss, A.J., Hawley, K. M. (2005). Youth psychotherapy outcome research: A review and critique of the evidence base. Annual Review Psychology, 56, 337-363. doi:10.1146/annurev.psych.55.090902.141449

Weisz, J. R., Southam-Gerow, M. A., Gordis, E. B., Connor-Smith, J. K., Chu, B. C., Langer, D. A., McLeod, B. D., Jensen-Doss, A., Updegraff, A., \& Weiss, B. (2009). Cognitivebehavioral therapy versus usual clinical care for youth depression: An initial test of transportability to community clinics and clinicians. Journal of Consulting and Clinical Psychology, 77, 383-396. doi:10.1037/a0013877 
Wood, J. J., Chiu, A.W., Hwang, W.-C., Jacobs, J., \& Ifekwunigwe, M. (2008). Adapting cognitive-behavioral therapy for Mexican American students with anxiety disorders: Recommendations for school psychologists. School Psychology Quarterly, 23, 515-532. doi:10.1037/1045-3830.23.4.515

Wong, D. F. K. (2008). Cognitive behavioral treatment groups for people with chronic depression in Hong Kong: A randomized wait-list control design. Depression and Anxiety, 25, 142-148. doi:10.1002/da.20286 


\section{Acknowledgements}

We are grateful to the children and parents for their participation in this study. We thank the staff at the Child Assessment Service, Department of Health, Hong Kong for their generous help in developing the treatment program, designing worksheets and treatment materials, implementing the treatment program, and doing diagnostic check of parent interviews. We thank Raymond Chan and Frendi Li for their clinical advice in program development and implementation, James Ho and Louise Pang for their help in translating assessment tools, and Karen Ravn, Lester Tong, and the reviewers for their critiques of earlier drafts. We thank Philip Kendall for his permission to adapt the Coping Cat program for use in a Chinese community, and Catherine Lam and Joseph Lau for their support allowing us to conduct this study in a clinic of the Child Assessment Service in Hong Kong. 
Table 1: Fit Indices for Hypothesized Models in the Present Study and Published Studies

\begin{tabular}{|c|c|c|c|c|c|c|c|c|c|}
\hline & & Study & $\chi^{2}$ & $d f$ & $p$ & NFI & NNFI & CFI & RMSEA \\
\hline Model 3: & Children & Spence & 1394 & 650 & $<.001$ & 0.94 & 0.97 & 0.97 & \\
\hline 6 correlated & & Present & 994 & 650 & $<.001$ & 0.97 & 1.00 & 1.00 & \\
\hline \multirow[t]{2}{*}{ factors } & Parents & Nauta & 3269 & 650 & $<.001$ & 0.89 & 0.90 & 0.91 & 0.08 \\
\hline & & Present & 1071 & 650 & $<.001$ & 0.98 & 1.00 & 1.00 & $<0.01$ \\
\hline Model 4: & Children & Spence & 1497 & 659 & $<.001$ & 0.94 & 0.96 & 0.96 & \\
\hline 6 correlated \& & & Present & \multicolumn{7}{|c|}{ Improper solution due to zero disturbance of Factor GAD } \\
\hline 1 higher-order & Parents & Nauta & \multicolumn{7}{|c|}{ Improper solution due to non-positive PSI } \\
\hline factors & & Present & 1137 & 659 & $<.001$ & 0.97 & 1.00 & 1.00 & $<0.01$ \\
\hline Model 5: & Children & Spence & \multicolumn{7}{|c|}{ Spence's study did not examine Model 5} \\
\hline 5 correlated \& & & Present & 1035 & 659 & $<.001$ & 0.97 & 1.00 & 1.00 & \\
\hline 1 higher-order & Parents & Nauta & 3392 & 660 & $<.001$ & 0.89 & 0.90 & 0.90 & \\
\hline factors & & Present & 1157 & 660 & $<.001$ & 0.97 & 1.00 & 1.00 & $<0.01$ \\
\hline
\end{tabular}

Note: $\mathrm{NFI}=$ norm fit index; NNFI = non-normed fit index; CFI = comparative fit index;

RMSEA $=$ root mean square error of approximation. 
Table 2: Means (Standard Deviation) of Outcome Measures for the CBT and Waitlist Condition and F-Statistics for Condition X Time (Time 1 vs. Time 2) Interaction

\begin{tabular}{|c|c|c|c|c|c|}
\hline & & CBT & Waitlist & F-Statistic & Partial $E t a^{2}$ \\
\hline \multicolumn{6}{|l|}{ Treatment Outcomes } \\
\hline \multicolumn{6}{|c|}{ Anxiety Symptoms } \\
\hline \multirow[t]{2}{*}{ SCAS } & Time 1 & $34.3(11.5)$ & $37.0(13.9)$ & $14.5 * * *$ & .27 \\
\hline & Time 2 & $24.6(10.5)$ & $38.8(13.7)$ & & \\
\hline \multirow[t]{2}{*}{ PSCAS } & Time 1 & $33.0(10.7)$ & $35.2(10.7)$ & $4.7^{*}$ & .11 \\
\hline & Time 2 & $28.8(10.3)$ & $36.5(11.0)$ & & \\
\hline \multicolumn{6}{|l|}{ Global Affect } \\
\hline \multirow[t]{2}{*}{ PANAS-NA } & Time 1 & $42.1(14.6)$ & $44.1(9.9)$ & 1.2 & .03 \\
\hline & Time 2 & $34.5(8.5)$ & $42.1(9.7)$ & & \\
\hline \multirow[t]{2}{*}{ PANAS-PA } & Time 1 & $38.1(10.8)$ & $37.6(5.8)$ & 3.7 & .08 \\
\hline & Time 2 & $38.9(6.6)$ & $35.7(7.7)$ & & \\
\hline \multicolumn{6}{|l|}{ Potential Mediators } \\
\hline \multicolumn{6}{|c|}{ Anxiety Cognition } \\
\hline \multirow[t]{2}{*}{ NASSQ } & Time 1 & $28.4(10.8)$ & $27.9(8.5)$ & $5.1 *$ & .11 \\
\hline & Time 2 & $21.5(9.8)$ & $28.8(12.0)$ & & \\
\hline \multicolumn{6}{|l|}{ Coping } \\
\hline \multirow[t]{2}{*}{ CQ-C } & Time 1 & $2.69(1.04)$ & $3.30(1.36)$ & $18.0 * * *$ & .31 \\
\hline & Time 2 & $4.29(1.3)$ & $2.97(1.3)$ & & \\
\hline \multirow[t]{2}{*}{ CQ-P } & Time 1 & $2.57(0.98)$ & $2.72(1.19)$ & $11.3 * * *$ & .22 \\
\hline & Time 2 & $4.08(1.2)$ & $3.03(1.1)$ & & \\
\hline
\end{tabular}

Note: p-levels: $* \mathrm{p}<.05, * * \mathrm{p}<.01, * * * \mathrm{p}<.001$ 


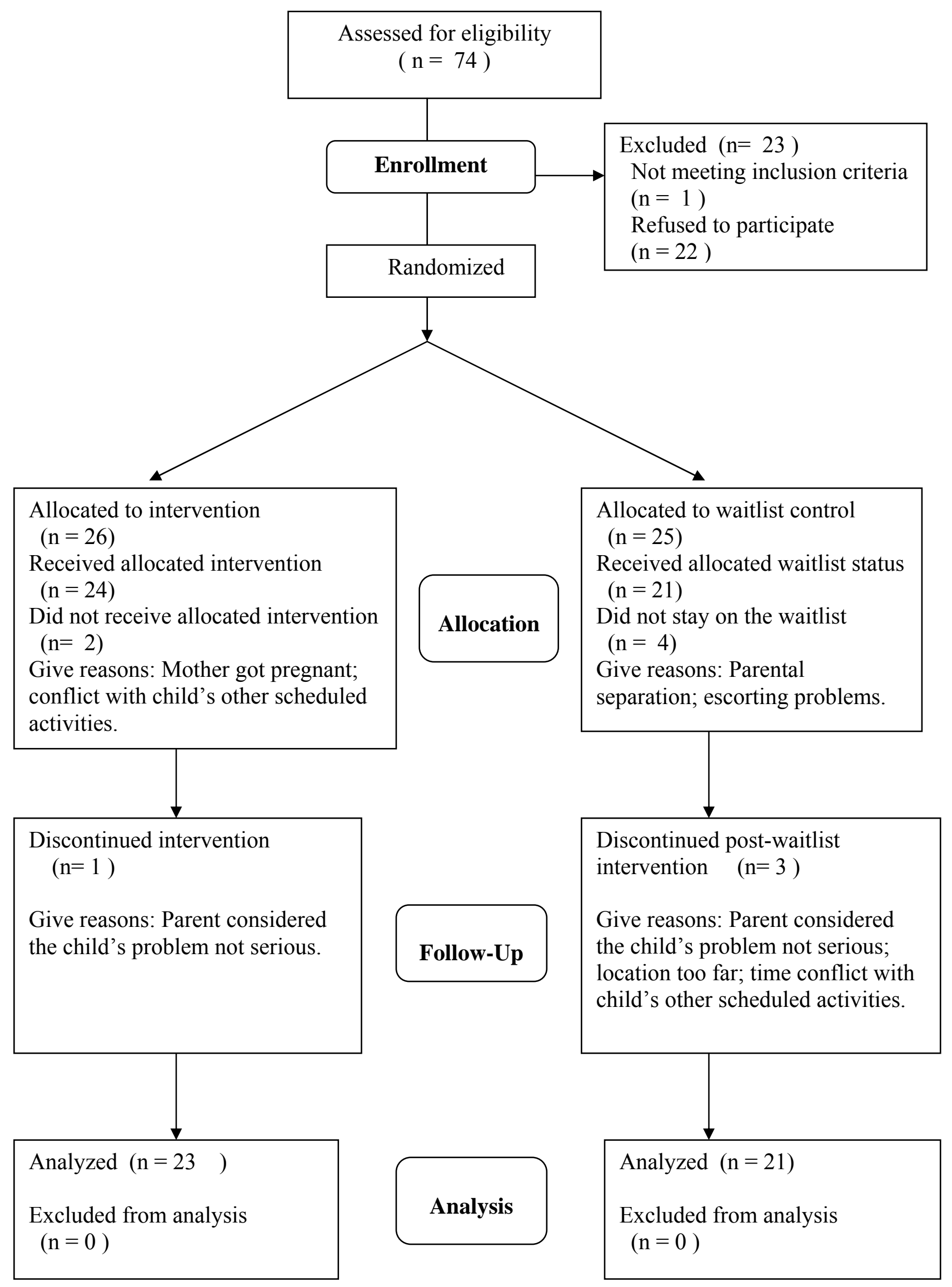

Figure 1. The CONSORT Flow Chart. 


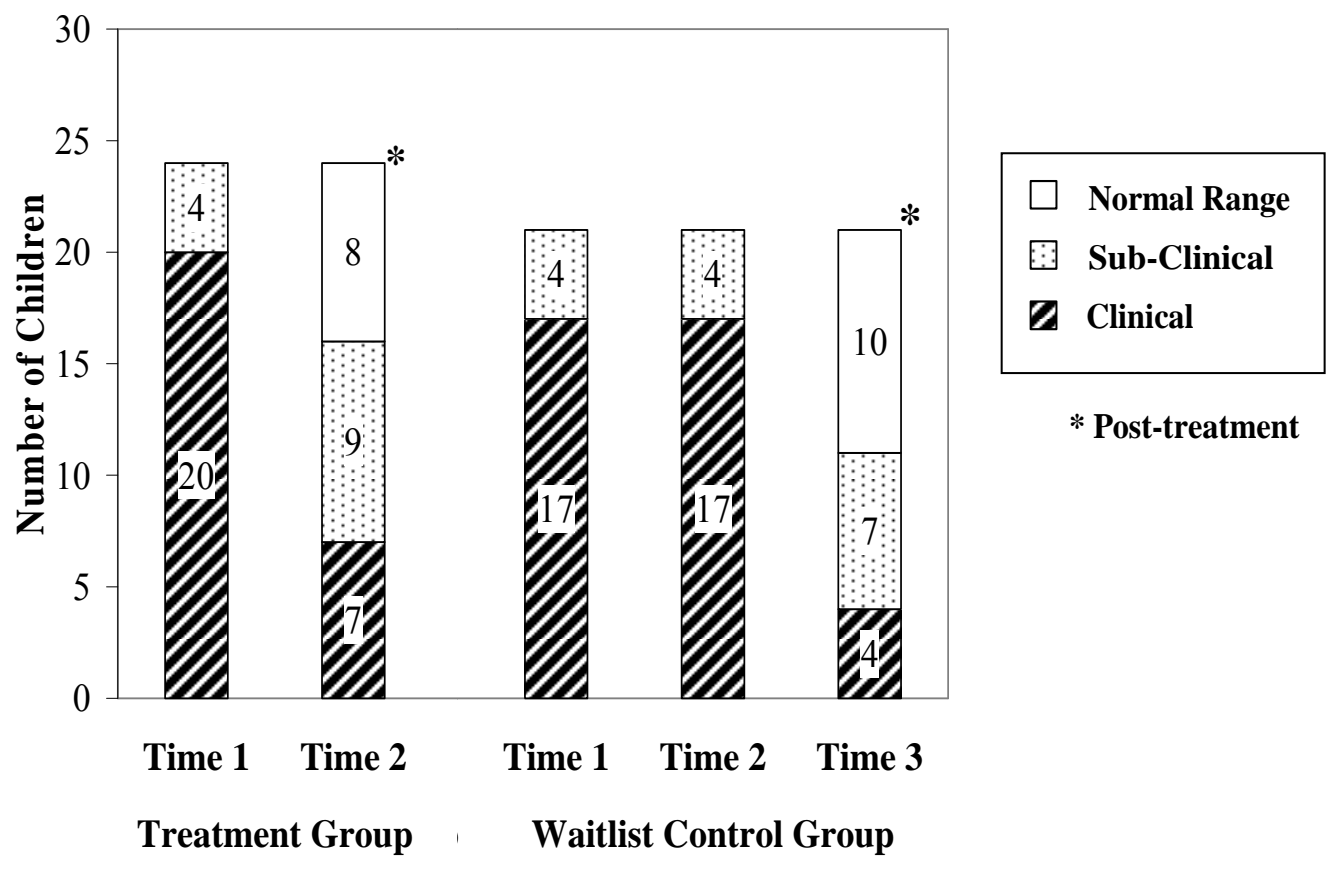

Figure 2. Diagnostic Status of Children in the Treatment vs. Waitlist Condition before and after Treatment 


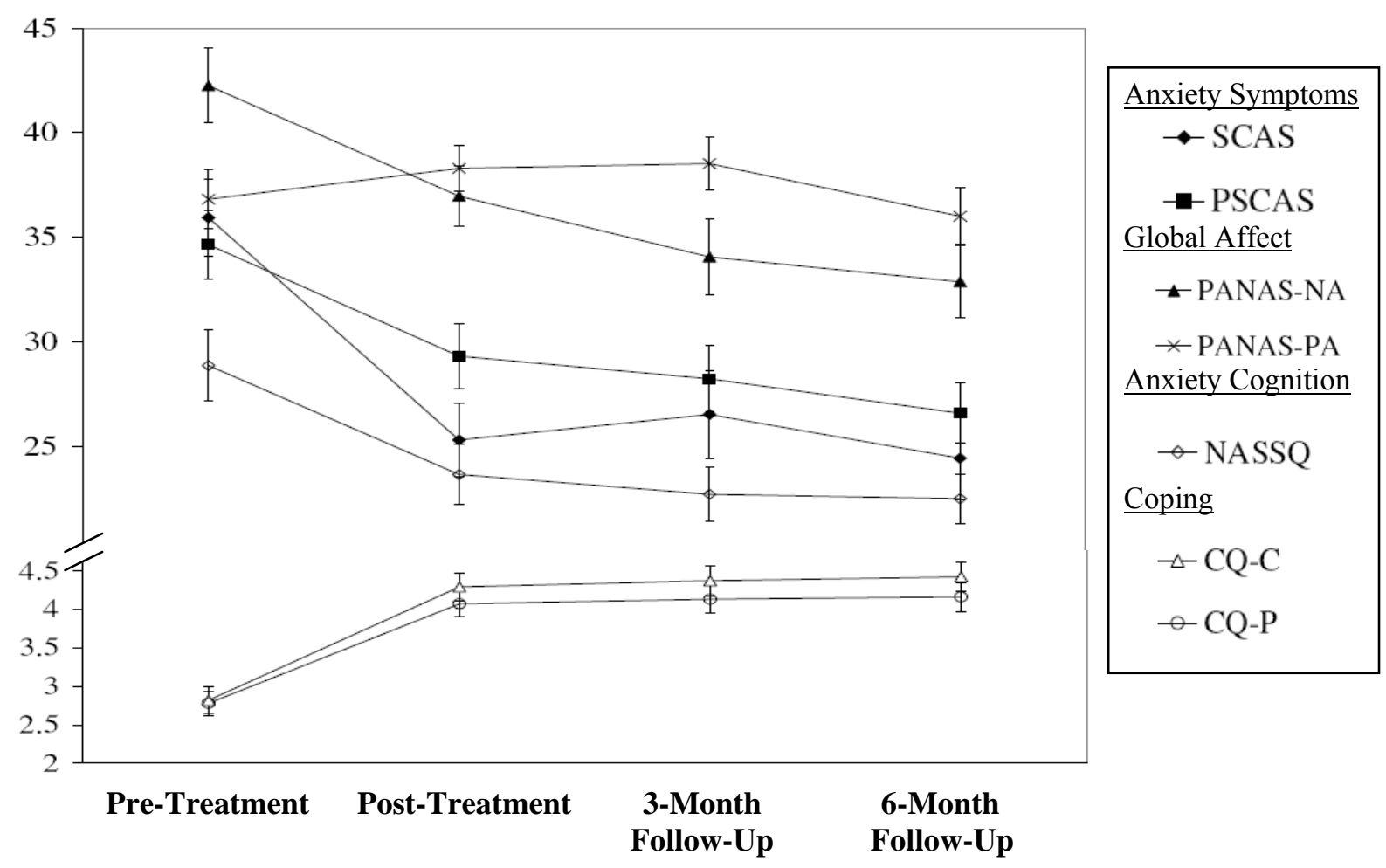

Figure 3. Outcome Measures on Children Pre- and Post-Treatment (immediate, 3-month and 6-month follow-up) 
(I) Direct Effect

\begin{tabular}{|c|c|c|}
\hline $\begin{array}{c}\text { Treatment } \\
\text { Status }\end{array}$ & $-14.18^{* * *}$ & SCAS \\
\cline { 3 - 4 }
\end{tabular}

(II) Mediation

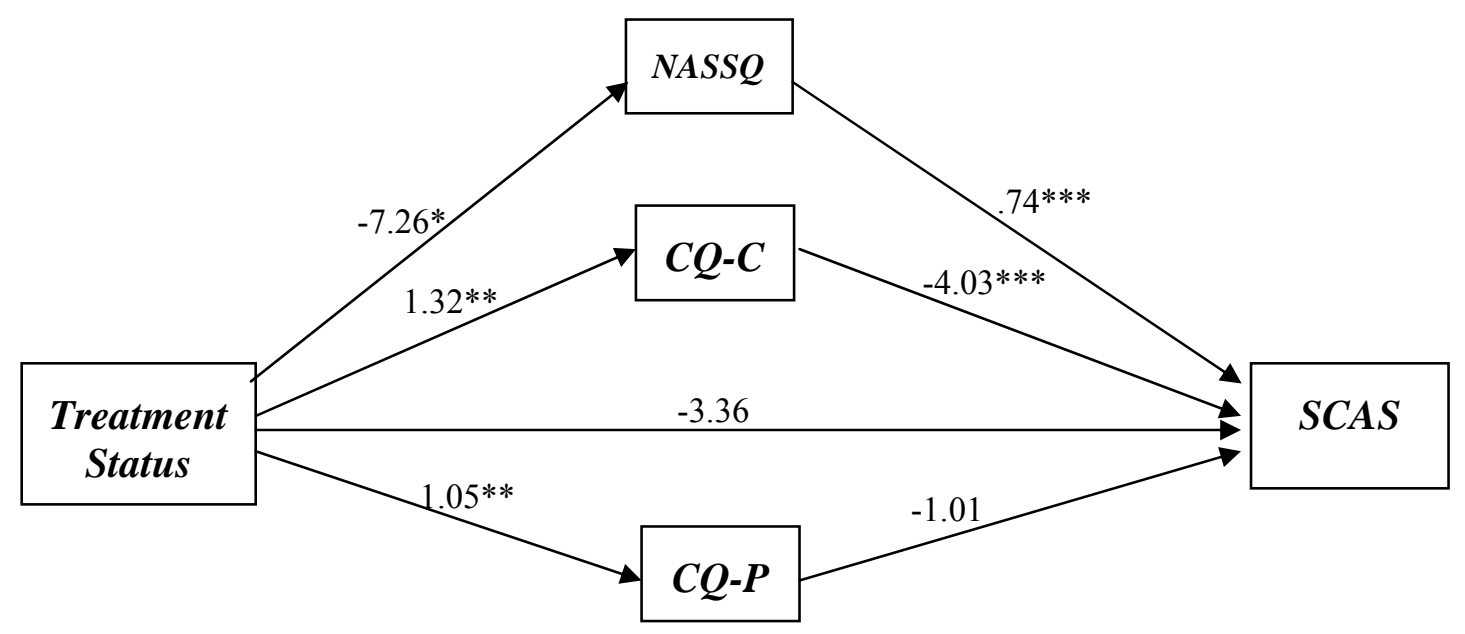

Figure 4. Path coefficients of the multiple mediation model for SCAS as outcome measure.

$\left({ }^{*} p<.05,{ }^{* *} p<.01,{ }^{* * *} p<.001\right)$ 
(I) Direct Effect

\begin{tabular}{|c|c|c|}
\hline $\begin{array}{c}\text { Treatment } \\
\text { Status }\end{array}$ & $-7.69^{*}$ & PSCAS \\
\cline { 3 - 4 }
\end{tabular}

(II) Mediation

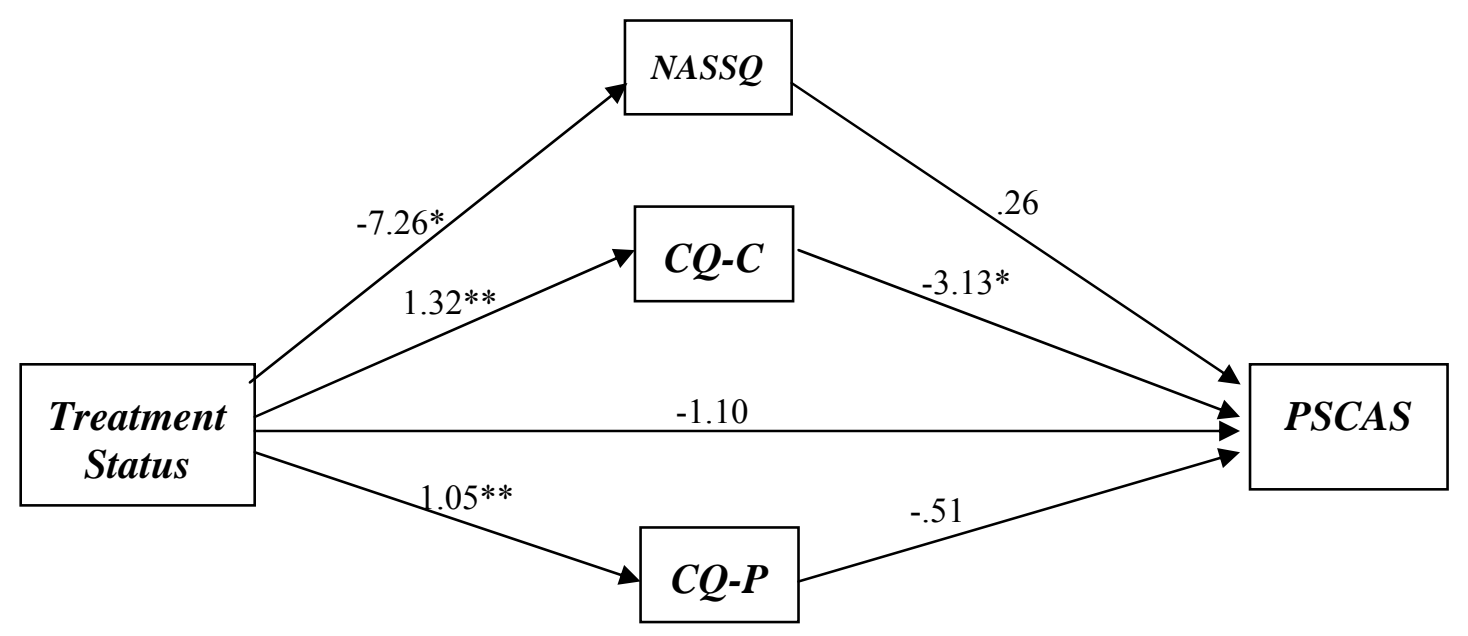

Figure 5. Path coefficients of the multiple mediation model for PSCAS as outcome measure $\left({ }^{*} p<.05,{ }^{* *} p<.01\right)$ 